\title{
Charged-Current Neutrino-Nucleus Scattering off the Even Molybdenum Isotopes
}

\author{
E. Ydrefors and J. Suhonen \\ Department of Physics, University of Jyväskylä, P.O. Box 35 (YFL), 40114 Jyväskylä, Finland \\ Correspondence should be addressed to E. Ydrefors, emanuel.ydrefors@jyu.fi
}

Received 5 July 2012; Revised 19 October 2012; Accepted 6 November 2012

Academic Editor: Gian Luigi Fogli

Copyright (C) 2012 E. Ydrefors and J. Suhonen. This is an open access article distributed under the Creative Commons Attribution License, which permits unrestricted use, distribution, and reproduction in any medium, provided the original work is properly cited.

Neutrinos from supernovae constitute important probes of both the currently unknown supernova mechanisms and of neutrino properties. Reliable information about the nuclear responses to supernova neutrinos is therefore crucial. In this work, we compute the cross sections for the charged-current neutrino-nucleus scattering off the even-even molybdenum isotopes. The nuclear responses to supernova neutrinos are subsequently calculated by folding the cross sections with a Fermi-Dirac distribution.

\section{Introduction}

Studies of neutrino-nucleus interactions with neutrinos of low and intermediate incoming energies (i.e., neutrino energies in the range up to a few hundred $\mathrm{MeV}$ ) are of great importance for the disentanglement of various unresolved questions in astrophysics, particle physics, and nuclear physics [1, 2]. Supernovae (type II) constitute the inevitable deaths of very massive stars initiated by the collapse of their iron cores. For the details of supernova physics, see for example, [3-5]. In a supernova event, about $99 \%$ of the explosion energy is emitted as neutrinos of all flavors. A future detection of neutrinos from a coming supernova would therefore provide a wealth of valuable information both on the currently unknown supernova mechanisms and the associated nucleosynthesis of heavy elements. A highstatistics observation of the neutrino signal from the next nearby supernova could in addition provide important information on unknown neutrino properties (see e.g., [6]). It has for example, been shown by simulations [7] that the signal produced by supernova neutrinos in a large-scale detector such as the IceCube [8] probably could be used to disentangle the important question of normal-or-inverted neutrino-mass hierarchy. As has been proposed by Volpe $[9,10]$, charged-current neutrino-nucleus scattering with neutrinos from low-energy 
neutrino beams could in the future be exploited for spectroscopy of the virtual states involved in neutrinoless double-beta decay (see e.g., [11]) and consequently constrain theoretical predictions for the associated nuclear matrix elements.

Neutrinos from astrophysical sources can be detected by Earth-bound detectors by using charged-current and/or neutral-current interactions in nuclei [12]. For a recent review on experimental methods for detection of supernova neutrinos, see [13]. One planned possibility for measurements of astrophysical neutrinos (solar and supernova neutrinos) is the MOON (Mo Observatory Of Neutrinos) experiment [14].

In this paper, we perform theoretical calculations of the cross sections for the chargedcurrent neutrino-nucleus scattering off the stable even-even molybdenum isotopes for neutrino energies, which are relevant for supernova neutrinos. We also present results for the averaged cross sections obtained by folding the cross sections with a Fermi-Dirac distribution. The calculations are based on the general theory for the treatment of semileptonic processes in nuclei, which was first introduced by O'Connell et al. [15]. This theory is discussed comprehensively in [16]. In the present calculations, the initial and final nuclear states are constructed by using the proton-neutron quasiparticle random-phase approximation (pnQRPA, see e.g., [17]).

The nuclear-structure dependence of the $v$-nucleus cross sections is contained in the reduced nuclear matrix elements of various one-body operators, which depend on the momentum transfer. With an increasing number of nuclear final states, the calculations of these matrix elements are obviously increasingly time consuming. Therefore, in [18] we introduced a fast method for the calculation of the involved matrix elements, which is based on the barycentric Lagrange interpolation [19]. This method is adopted in this work and it is shown that the obtained results are in very good agreement with exact calculations.

This paper is organized as follows. In Section 2 we outline the theoretical framework for calculations of charged-current neutrino-nucleus cross sections. First the pnQRPA is introduced. We then briefly summarize the formalism for computations of charged-current neutrino-nucleus scattering off nuclei. Then, in Section 3 we present our results. Finally, in Section 4 we draw the conclusions.

\section{Theory}

\section{1. pnQRPA}

In this work, the proton-neutron quasiparticle random-phase approximation (pnQRPA) is adopted to construct the nuclear final and initial states. In this section, we therefore briefly summarize the formalism of the pnQRPA. For a more detailed treatment, the reader is referred to $[17,20]$.

The nuclear Hamiltonian for a general two-body residual interaction $V$ can be expressed in the form

$$
H=\sum_{\alpha} \varepsilon_{a} c_{\alpha}^{\dagger} c_{\alpha}+\frac{1}{4} \sum_{\alpha \beta \gamma \delta} \bar{v}_{\alpha \beta \gamma \beta} c_{\alpha}^{\dagger} c_{\beta}^{\dagger} c_{\delta} c_{\gamma}
$$

where the index $a$ contains the single-particle quantum numbers $n_{a}, l_{a}$, and $j_{a}$ and $\alpha$ holds in addition the magnetic quantum number $m_{\alpha}$. Here $c_{\alpha}^{\dagger}$ is the particle creation operator and $c_{\alpha}$ denotes the corresponding particle annihilation operator. In (2.1) $\varepsilon_{a}$ are single-particle 
energies and the antisymmetric two-body matrix elements are defined as $\bar{v}_{\alpha \beta \gamma \beta}=\langle\alpha \beta|V| \gamma \delta\rangle-$ $\langle\alpha \beta|V| \delta \gamma\rangle$.

The pairing correlations in this work are taken into account by adopting the BCS (Bardeen-Cooper-Schrieffer) theory. The starting point in the BCS approach is the variational ansatz for the BCS vacuum

$$
|\mathrm{BCS}\rangle=\prod_{\alpha>0}\left(u_{a}-v_{a} c_{\alpha}^{\dagger} \widetilde{c}_{\alpha}^{\dagger}\right)|\mathrm{HF}\rangle
$$

where $|\mathrm{HF}\rangle$ is the Hartree-Fock vacuum and $\tilde{c}_{\alpha}$ denotes the time-reversed particle annihilation operator, which is defined as $\tilde{c}_{\alpha}=(-1)^{j_{a}+m_{\alpha}} C_{-\alpha}$ where $-\alpha=\left(a,-m_{\alpha}\right)$. The variational parameters $u_{a}$ and $v_{a}$ in (2.2) are obtained by solving the BCS equations [17].

The quasiparticles subsequently are defined via the Bogoliubov-Valatin transformation

$$
\begin{aligned}
& a_{\alpha}^{\dagger}=u_{a} c_{\alpha}^{\dagger}+v_{a} \tilde{c}_{\alpha}, \\
& \tilde{a}_{\alpha}=u_{a} \tilde{c}_{\alpha}-u_{a} c_{\alpha}^{\dagger} .
\end{aligned}
$$

By using this transformation the nuclear Hamiltonian (2.1) can be cast into the form

$$
H=H_{11}+H_{02}+H_{20}+H_{13}+H_{31}+H_{04}+H_{40}
$$

where the term $H_{i j}$ is proportional to a normal ordered product of $i$ quasiparticle creation operators and $j$ quasiparticle annihilation operators.

In practice, the parameters of the BCS calculation are adjusted to reproduce the experimental pairing gaps for the reference even-even nucleus under consideration. In this work, the empirical pairing gaps have been deduced from the three-point formulae (see e.g., [17])

$$
\begin{gathered}
\Delta_{\mathrm{p}}=\frac{1}{4}(-1)^{Z+1}\left[S_{\mathrm{p}}(A+1, Z+1)-2 S_{\mathrm{p}}(A, Z)+S_{\mathrm{p}}(A-1, Z-1)\right], \\
\Delta_{\mathrm{n}}=\frac{1}{4}(-1)^{A-Z+1}\left[S_{\mathrm{n}}(A+1, Z)-2 S_{\mathrm{n}}(A, Z)+S_{\mathrm{n}}(A-1, Z)\right],
\end{gathered}
$$

where $S_{\mathrm{p}}(A, Z)\left(S_{\mathrm{n}}(A, Z)\right)$ is the proton (neutron) separation energy for the nucleus $(A, Z)$ having mass number $A$ and proton number $Z$.

In the pnQRPA, the states of the odd-odd nucleus subsequently are constructed by coupling two-quasiparticle operators to good angular momentum $J_{\omega}$ and parity $\pi_{\omega}$. The state vector corresponding to the excitation $\omega=\left(J_{\omega}, \pi_{\omega}, k_{\omega}\right)$ then is defined by

$$
|\omega\rangle=Q_{\omega}^{\dagger}|\mathrm{pnQRPA}\rangle,
$$


where $|p n Q R P A\rangle$ denotes the pnQRPA vacuum and the additional quantum number $k_{\omega}$ enumerates the states with the same angular momentum and parity. Here the pnQRPA creation operator $Q_{\omega}^{\dagger}$ is given by

$$
Q_{\omega}^{\dagger}=\sum_{p n}\left(X_{p n}^{\omega}\left[a_{p}^{\dagger} a_{n}^{\dagger}\right]_{J_{\omega} M_{\omega}}+Y_{p n}^{\omega}\left[\tilde{a}_{p} \tilde{a}_{n}\right]_{J_{\omega} M_{\omega}}\right)
$$

where the sum runs over all possible proton-neutron configurations in the adopted valence space. The pnQRPA equations can then be written in the matrix form [17]

$$
\left(\begin{array}{cc}
\mathbf{A} & \mathbf{B} \\
-\mathbf{B}^{*} & -\mathbf{A}^{*}
\end{array}\right)\left(\begin{array}{l}
\mathbf{X}^{\omega} \\
\mathbf{Y}^{\omega}
\end{array}\right)=E_{\omega}\left(\begin{array}{l}
\mathbf{X}^{\omega} \\
\mathbf{Y}^{\omega}
\end{array}\right)
$$

where $E_{\omega}$ denotes the pnQRPA energy of the phonon $\omega$. In (2.8) $\mathbf{A}$ is the well-known pnQTDA matrix and the matrix $\mathbf{B}$ contains the induced ground-state correlations. Matrices $\mathbf{A}$ and $\mathbf{B}$ contain both a particle-particle and a particle-hole channel of the proton-neutron twobody interaction. As is customary [11] also in this work the particle-particle and particle-hole channels are scaled by phenomenological constants $g_{\mathrm{pp}}$ and $g_{\mathrm{ph}}$, respectively. This is done for each multipole $J^{\pi}$ separately. For more details on the scaling of the pnQRPA Hamiltonian, see $[20,21]$. In the present computations, these parameters are tuned for the $1^{+}$multipole only by using experimental observables such as beta-decay rates, the energy of the giant GamowTeller resonance, and experimental energies of the low-lying states in the odd-odd nucleus under consideration.

\subsection{Charged-Current Neutrino-Nucleus Scattering}

In this work, we consider charged-current neutrino and antineutrino scatterings off a nucleus $(A, Z)$ with mass number $A$ and proton number $Z$, that is, neutrino-induced reactions of either the form

$$
v_{l}+(A, Z) \longrightarrow(A, Z+1)+l^{-},
$$

or

$$
\bar{v}_{l}+(A, Z) \longrightarrow(A, Z-1)+l^{+},
$$

for the lepton flavors $l=\mathrm{e}, \mu, \tau$. The Feynman diagram for the $v$-nucleus scattering that proceed, via an exchange of a $W^{+}$boson is shown in Figure 1. In the figure $k_{\mu},\left(k_{\mu}^{\prime}\right)$ represents the four-momentum of the incoming (outgoing), lepton and $p_{\mu}$ and $p_{\mu}^{\prime}$ are the four-momenta of the initial and final nuclei. The antineutrino reaction $(2.10)$, which is mediated by a $W^{-}$ boson, can be obtained via charge-conjugation operation on Figure 1 and therefore is not displayed here.

In this work, we employ conventions that are similar to the ones used in [22]. We consequently define the covariant and contravariant four-vectors as $a_{\mu}=\left(a_{0},-\mathbf{a}\right)$ and $a^{\mu}=$ $g^{\mu v} a_{v}=\left(a_{0}, \mathbf{a}\right)$, respectively, with the metric $g^{\mu v}=g_{\mu v}=\operatorname{diag}(1,-1,-1,-1)$. We thus write 


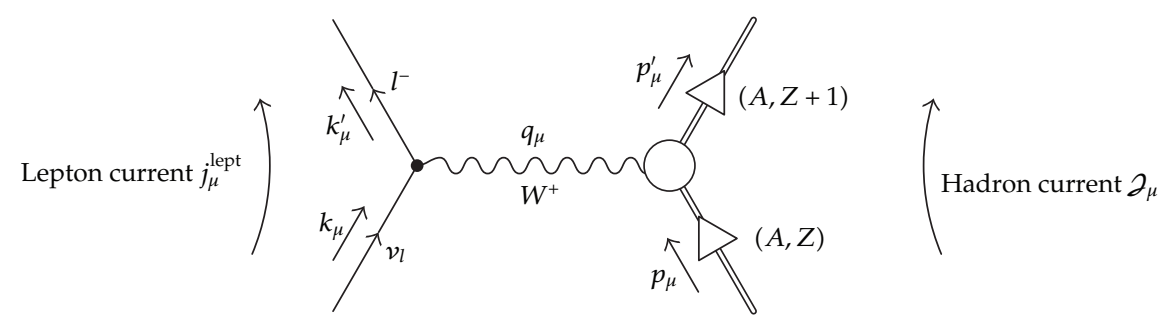

Figure 1: Charged-current neutrino scattering off a nucleus $(A, Z)$ via the exchange of a $W^{+}$boson. The transferred four momentum is $q_{\mu}=k_{\mu}^{\prime}-k_{\mu}=p_{\mu}-p_{\mu}^{\prime}$.

$k_{\mu}=\left(E_{\mathbf{k}},-\mathbf{k}\right)$ and $k_{\mu}^{\prime}=\left(E_{\mathbf{k}^{\prime}},-\mathbf{k}^{\prime}\right)$, where $E_{\mathbf{k}}$ and $E_{\mathbf{k}^{\prime}}$ are the energies and $\mathbf{k}$ and $\mathbf{k}^{\prime}$ denote the three-momenta of the incoming and outgoing leptons. The four-momenta of the initial and final nuclear states take similarly the forms $p_{\mu}=\left(E_{\mathbf{p}},-\mathbf{p}\right)$ and $p_{\mu}^{\prime}=\left(E_{\mathbf{p}^{\prime},}-\mathbf{p}^{\prime}\right)$, respectively.

For low-energy neutrinos, such as the supernova neutrinos that are considered in this work, the transferred four momentum is small compared to the mass of the gauge boson $\left(W^{+}\right.$and $W^{-}$resp.), that is, $Q^{2}=-q_{\mu} q^{\mu} \ll M_{W}^{2}$. The process in Figure 1 can consequently be treated in lowest order in the coupling constant $G$. The effective Hamiltonian density can then be written for the neutrino-induced reaction (2.9) in the current-current form

$$
H_{\mathrm{eff}}^{v}(\mathbf{r})=\frac{G}{\sqrt{2}} j_{\mu}^{(-), \text {lept }}(\mathbf{r}) \partial^{(+), \mu}(\mathbf{r}), \quad \mathbf{r}=(x, y, z)
$$

where $j_{\mu}^{(-), \text {lept }}(\mathbf{r})$ represents the charged-current lepton current and $\partial^{(+), \mu}(\mathbf{r})$ is the hadron current. For charge-changing interactions, the coupling constant $G$ is given by $G=G_{\mathrm{F}} \cos \theta_{\mathrm{C}}$ where $G_{\mathrm{F}}=1.1644 \times 10^{-5}, \mathrm{GeV}$ denotes the Fermi constant, and $\theta_{\mathrm{C}} \approx 13^{\circ}$ is the Cabibbo angle. For the antineutrino scattering, the effective Hamiltonian density similarly is given by

$$
H_{\mathrm{eff}}^{\bar{v}}(\mathbf{r})=\frac{G}{\sqrt{2}} j_{\mu}^{(+), \text {lept }}(\mathbf{r}) \partial^{(-), \mu}(\mathbf{r})
$$

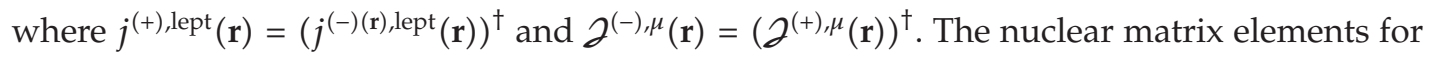
both the considered processes then take the form

$$
\begin{aligned}
\left\langle f\left|H_{\mathrm{eff}}^{v / \bar{v}}\right| i\right\rangle & =\frac{G}{\sqrt{2}} \int \mathrm{d}^{3} \mathbf{r}\left\langle f_{l}\left|j_{\mu}^{\text {lept }}(\mathbf{r})\right| f_{\nu_{l}}\right\rangle\left\langle f\left|\partial^{\mu}(\mathbf{r})\right| i\right\rangle \\
& =\frac{G}{\sqrt{2}} \int \mathrm{d}^{3} \mathbf{r e}^{-\mathrm{iq} \cdot \mathbf{r}} l_{\mu}\left\langle f\left|\partial^{\mu}(\mathbf{r})\right| i\right\rangle \\
& =\frac{G}{\sqrt{2}} \int \mathrm{d}^{3} \mathrm{re}^{-\mathrm{iq} \cdot \mathbf{r}}\left[l_{0}\left\langle f\left|\partial_{0}\right| i\right\rangle-1 \cdot\langle f|\partial(\mathbf{r})| i\rangle\right],
\end{aligned}
$$

where $f_{l}=l^{-}\left(l^{+}\right)$and $f_{v_{l}}=v_{l}\left(\bar{v}_{l}\right)$ for neutrino (antineutrino) scattering. We have here defined $\partial^{\mu}(\mathbf{r})=\left(\partial_{0}(\mathbf{r}), \mathbf{J}(\mathbf{r})\right)=\partial^{( \pm), \mu}(\mathbf{r})$, and $j_{\mu}^{\text {lept }}(\mathbf{r})=j^{( \pm), \mu}(\mathbf{r})$. This should be understood such that the appropriate hadron and lepton currents for the process under consideration are 
combined according to (2.11) and (2.12), respectively. In (2.13) we have moreover introduced the lepton matrix element

$$
l_{\mu}=\mathrm{e}^{\mathrm{iq} \cdot \mathbf{r}}\left\langle f_{l}\left|j_{\mu}^{\text {lept }}(\mathbf{r})\right| f_{v}\right\rangle .
$$

Here the hadron currents $2^{( \pm), \mu}(\mathbf{r})$ can be decomposed into vector $(\mathrm{V})$ and axial-vector $(\mathrm{A})$ pieces according to

$$
\partial^{( \pm), \mu}(\mathbf{r})=2^{( \pm), \mathrm{V}, \mu}(\mathbf{r})-\partial^{( \pm), \mathrm{A}, \mu}(\mathbf{r})
$$

At the origin $(\mathbf{r}=0)$, the single-nucleon matrix elements of the currents $\partial^{(+), V, \mu}$ and $\partial^{(+), A, \mu}$ are given by

$$
\begin{aligned}
& \mathrm{p}\left\langle\mathbf{p}^{\prime} \sigma^{\prime}\left|J^{(+), \mathrm{v}, \mu}(0)\right| \mathbf{p} \sigma\right\rangle_{\mathrm{n}}=\frac{\bar{u}\left(\mathbf{p}^{\prime}, \sigma^{\prime}\right)}{V}\left[F_{1}^{\mathrm{CC}}\left(Q^{2}\right) \gamma^{\mu}-\mathrm{i} \frac{F_{2}^{\mathrm{CC}}\left(Q^{2}\right)}{2 m_{\mathrm{N}}} \sigma^{\mu v} q_{v}\right] u(\mathbf{p}, \sigma), \\
& \mathrm{p}\left\langle\mathbf{p}^{\prime} \sigma^{\prime}\left|J^{(+), \mathrm{A}, \mu}(0)\right| \mathbf{p} \sigma\right\rangle_{\mathrm{n}}=\frac{\bar{u}\left(\mathbf{p}^{\prime}, \sigma^{\prime}\right)}{V}\left[F_{\mathrm{A}}^{\mathrm{CC}}\left(Q^{2}\right) \gamma_{5} \gamma^{\mu}-F_{\mathrm{P}}^{\mathrm{CC}}\left(Q^{2}\right) \gamma_{5} q^{v}\right] u(\mathbf{p}, \sigma),
\end{aligned}
$$

where $|\mathbf{p} \sigma\rangle_{\mathrm{p}(\mathrm{n})}$ denotes the state vector of a proton (neutron) having the three momentum $\mathbf{p}$ and spin projection $\sigma$ and $m_{\mathrm{N}}$ is the nucleon mass. The corresponding one-nucleon matrix elements for the nuclear current $2^{(-), \mu}(\mathbf{r})$ are related to the ones of $\partial^{(+), \mu}(\mathbf{r})$ through the charge symmetries [23]

$$
\begin{aligned}
& { }_{\mathrm{n}}\left\langle\mathbf{p}^{\prime} \sigma^{\prime}\left|J^{(-), \mathrm{v}, \mu}(0)\right| \mathbf{p} \sigma\right\rangle_{\mathrm{p}}={ }_{\mathrm{p}}\left\langle\mathbf{p}^{\prime} \sigma^{\prime}\left|J^{(+), \mathrm{v}, \mu}(0)\right| \mathbf{p} \sigma\right\rangle_{\mathrm{n}^{\prime}} \\
& { }_{\mathrm{n}}\left\langle\mathbf{p}^{\prime} \sigma^{\prime}\left|J^{(-), \mathrm{A}, \mu}(0)\right| \mathbf{p} \sigma\right\rangle_{\mathrm{p}}={ }_{\mathrm{p}}\left\langle\mathbf{p}^{\prime} \sigma^{\prime}\left|J^{(+), \mathrm{A}, \mu}(0)\right| \mathbf{p} \sigma\right\rangle_{\mathrm{n}} .
\end{aligned}
$$

By the conservation of the vector current $(\mathrm{CVC})$, the vector form factors $F_{1,2}^{\mathrm{CC}}\left(Q^{2}\right)$ in (2.16) can be written in terms of the proton and neutron electromagnetic form factors $F_{1,2}^{\mathrm{EM} ; \mathrm{p}}\left(Q^{2}\right)$ and $F_{1,2}^{\mathrm{EM} ; \mathrm{n}}\left(Q^{2}\right)$ as (see e.g., [23])

$$
F_{1,2}^{\mathrm{CC}}\left(Q^{2}\right)=F_{1,2}^{\mathrm{EM} ; \mathrm{p}}\left(Q^{2}\right)-F_{1,2}^{\mathrm{EM} ; \mathrm{n}}\left(Q^{2}\right)
$$

In the present work, we use the electromagnetic form factors of [22]. The axial-vector form factor $F_{\mathrm{A}}^{\mathrm{CC}}\left(Q^{2}\right)$ in (2.16) is assumed to be of dipole form with the quenched static value $F_{\mathrm{A}}^{\mathrm{CC}}(0)=-1.0$. Moreover, the pseudoscalar form factor $F_{\mathrm{P}}^{\mathrm{CC}}\left(Q^{2}\right)$ in $(2.16)$ is obtained from the Goldberger-Treiman relation (see e.g., [16])

$$
F_{\mathrm{P}}^{\mathrm{CC}}\left(Q^{2}\right) \approx \frac{2 m_{\mathrm{N}}}{m_{\pi}^{2}+Q^{2}} F_{\mathrm{A}}^{\mathrm{CC}}\left(Q^{2}\right)
$$

where $m_{\pi}=139.57 \mathrm{MeV}$ [5] represents the mass of the charged pion. 
We assume in this work that the final and initial nuclear states have well-defined angular momenta $J_{f}$ and $J_{i}$ and parities $\pi_{f}$ and $\pi_{i}$, respectively. The double-differential cross section for the charged-current neutrino-nucleus scattering with transition from an initial nuclear state $i$ to a final nuclear state $f$ can then be written in the form (see e.g., [24])

$$
\left(\frac{\mathrm{d}^{2} \sigma_{i \rightarrow f}}{\mathrm{~d} \Omega \mathrm{d} E_{\mathrm{exc}}}\right)_{\nu / \bar{v}}=\frac{G^{2} F\left( \pm Z_{f}, E_{\mathbf{k}^{\prime}}\right)\left|\mathbf{k}^{\prime}\right| E_{\mathbf{k}^{\prime}}}{\pi\left(2 J_{i}+1\right)}\left(\sum_{J} \sigma_{\mathrm{CL}}^{J}+\sum_{J \geq 1} \sigma_{\mathrm{T}}^{J}\right),
$$

where

$$
\begin{aligned}
\sigma_{\mathrm{CL}}^{J}= & (1+a \cos \theta)\left|\left(J_{f}\left\|\mathcal{M}_{J}(q)\right\| J_{i}\right)\right|^{2} \\
& +\left(1+a \cos \theta-2 b \sin ^{2} \theta\right)\left|\left(J_{f}\left\|\mathcal{L}_{J}(q)\right\| J_{i}\right)\right|^{2} \\
& +\frac{E_{\mathrm{exc}}}{q}((1+a \cos \theta)+c) 2 \operatorname{Re}\left[\left(J_{f}\left\|\mathcal{L}_{J}(q)\right\| J_{i}\right)\left(J_{f}\left\|\mathcal{M}_{J}(q)\right\| J_{i}\right)^{*}\right], \\
\sigma_{\mathrm{T}}^{J}= & \left(1-a \cos \theta+b \sin ^{2} \theta\right)\left[\left|\left(J_{f}\left\|\tau_{J}^{\mathrm{mag}}(q)\right\| J_{i}\right)\right|^{2}+\left|\left(J_{f}\left\|\tau_{J}^{\mathrm{el}}(q)\right\| J_{i}\right)\right|^{2}\right] \\
& \mp \frac{\left(E_{\mathbf{k}}+E_{\mathbf{k}^{\prime}}\right)}{q}[((1-a \cos \theta)-c)] 2 \operatorname{Re}\left[\left(J_{f}\left\|\tau_{J}^{\mathrm{mag}}(q)\right\| J_{i}\right)\left(J_{f}\left\|\tau_{J}^{\mathrm{el}}(q)\right\| J_{i}\right)^{*}\right] .
\end{aligned}
$$

Here the excitation energy $E_{\text {exc }}$ of the final nuclear state is defined with respect to the initial nuclear state, that is, $E_{\mathrm{exc}}=E_{\mathrm{p}^{\prime}}-E_{\mathrm{p}}$. In the above expressions, we have introduced

$$
\begin{gathered}
a=\sqrt{1-\frac{m_{f}^{2}}{E_{\mathbf{k}^{\prime}}^{2}}}, \\
b=\frac{a^{2} E_{\mathbf{k}} E_{\mathbf{k}^{\prime}}}{q^{2}}, \\
c=\frac{m_{f}^{2}}{q E_{\mathbf{k}^{\prime}}}
\end{gathered}
$$

where the magnitude of the three-momentum transfer $q$ is given by

$$
q=|\mathbf{q}|=\sqrt{a^{2} E_{\mathbf{k}^{\prime}}^{2}+E_{\mathbf{k}}^{2}-a \cos \theta\left(2 E_{\mathbf{k}} E_{\mathbf{k}^{\prime}}\right)}
$$

and $m_{f}$ denotes the rest mass of the outgoing lepton. In (2.20), the function $F\left( \pm Z_{f}, E_{\mathbf{k}^{\prime}}\right)$, where $Z_{f}$ is the proton number of the final nucleus, takes into account the distortion of the outgoing lepton wave function due to the interaction with the (final) nucleus and the $+(-)$ sign is used in the case of neutrinos (antineutrinos). Furthermore, in (2.22) the minus sign is used for neutrinos and the plus sign for antineutrinos. 
For the treatment of final-state interactions, we use the method introduced by Engel in [25]. Consequently, we define the effective momentum of the outgoing lepton within the nucleus as

$$
k_{\mathrm{eff}}=\sqrt{E_{\mathrm{eff}}-m_{l}^{2}}
$$

where the effective energy is given by

$$
E_{\text {eff }}=E_{\mathbf{k}^{\prime}}-V_{C}(0)
$$

Here $V_{C}(0)$ is the value at the origin of the Coulomb potential produced by the final nucleus. In this work, we approximate the Coulomb field with that of a uniformly charged sphere and therefore $V_{C}(0)=-\alpha Z(3 / 2 R)$ where $Z=Z_{f}$ for leptons and $Z=-Z_{f}$ for antileptons and $R$ denotes the nuclear radius. In the region where $k_{\text {eff }}$ is small, that is, $k_{\text {eff }} R \ll 1$, one can, just as in beta-decay calculations, adopt a Fermi function of the form [26]

$$
F\left(Z, E_{\mathbf{k}^{\prime}}\right)=2\left(1+\gamma_{0}\right)\left(2\left|\mathbf{k}^{\prime}\right| R\right)^{-2\left(1-\gamma_{0}\right)} \mathrm{e}^{\pi \nu} \frac{\left|\Gamma\left(\gamma_{0}+\mathrm{i} v\right)\right|^{2}}{\Gamma\left(2 \gamma_{0}+1\right)^{2}}
$$

where

$$
\gamma_{0}=\sqrt{1-Z^{2} \alpha}, \quad v=\frac{Z \alpha E_{\mathbf{k}^{\prime}}}{\left|\mathbf{k}^{\prime}\right|}
$$

For larger values of the energy $E_{\mathbf{k}^{\prime}}$ of the final lepton, we employ in the present work the so-called modified effective momentum approximation (EMA) introduced in [25]. In this approximation, the absolute value of the three momentum and the energy of the outgoing lepton are replaced by the effective values given by (2.25) and (2.26), respectively. The effective energy and momentum are also used in the calculation of the three-momentum transfer $q$ defined by (2.24).

Guided by $[10,25]$, we adopt in the present calculations for the neutrino interaction (2.9) the Coulomb treatment which, gives the smallest cross section. For the antineutrino reaction (2.10), the final-state interactions decrease the cross section. Therefore, we choose in this case the Coulomb treatment (Fermi function or modified EMA), which gives the largest cross section.

\section{Results and Discussion}

In this section, we adopt the formalism presented in Section 2 to compute the cross sections for the charged-current neutrino and antineutrino scatterings off the even-even molybdenum isotopes.

The initial and final nuclear states are first constructed by using the pnQRPA (proton-neutron quasiparticle random-phase approximation) discussed in Section 2.1. In the calculations, the single-particle energies were generated by using the Coulomb-corrected 
Table 1: Cross sections for the charged-current neutrino-nucleus scattering off the even molybdenum nuclei in units of $10^{-42} \mathrm{~cm}^{2}$. Exponents are shown in parenthesis. In the last column, we also show for comparison the results for ${ }^{100}$ Mo calculated in [10].

\begin{tabular}{lcccccc}
\hline$E_{\mathbf{k}} / \mathrm{MeV}$ & ${ }^{92} \mathrm{Mo}$ & ${ }^{94} \mathrm{Mo}$ & ${ }^{96} \mathrm{Mo}$ & ${ }^{98} \mathrm{Mo}$ & ${ }^{100 \mathrm{Mo}}$ & ${ }^{100} \mathrm{Mo}[10]$ \\
\hline 5.0 & - & $3.16(-2)$ & $4.00(-1)$ & $1.02(0)$ & $2.83(0)$ & - \\
10.0 & $9.43(-2)$ & $1.97(0)$ & $4.45(0)$ & $7.51(0)$ & $1.38(1)$ & $0.68(1)$ \\
15.0 & $8.96(0)$ & $1.89(1)$ & $2.98(1)$ & $4.21(1)$ & $5.91(1)$ & $4.60(1)$ \\
20.0 & $4.75(1)$ & $8.13(1)$ & $1.19(2)$ & $1.59(2)$ & $2.13(2)$ & $2.09(2)$ \\
25.0 & $1.54(2)$ & $2.28(2)$ & $3.04(2)$ & $3.78(2)$ & $4.84(2)$ & $5.01(2)$ \\
30.0 & $3.27(2)$ & $4.48(2)$ & $5.70(2)$ & $6.73(2)$ & $8.57(2)$ & $9.04(2)$ \\
40.0 & $8.20(2)$ & $1.04(3)$ & $1.25(3)$ & $1.39(3)$ & $1.75(3)$ & $1.96(3)$ \\
50.0 & $1.58(3)$ & $1.94(3)$ & $2.25(3)$ & $2.43(3)$ & $3.02(3)$ & $3.02(3)$ \\
60.0 & $2.61(3)$ & $3.07(3)$ & $3.47(3)$ & $3.73(3)$ & $4.48(3)$ & $4.67(3)$ \\
70.0 & $3.78(3)$ & $4.33(3)$ & $4.82(3)$ & $5.15(3)$ & $6.04(3)$ & $6.80(3)$ \\
80.0 & $4.99(3)$ & $5.63(4)$ & $6.21(4)$ & $6.60(3)$ & $7.63(3)$ & $9.36(3)$ \\
\hline
\end{tabular}

Table 2: Cross sections for the charged-current antineutrino scattering off the even molybdenum nuclei in units of $10^{-42} \mathrm{~cm}^{2}$. Exponents are shown in parenthesis.

\begin{tabular}{lccccc}
\hline$E_{\mathbf{k}} / \mathrm{MeV}$ & ${ }^{92} \mathrm{Mo}$ & ${ }^{94} \mathrm{Mo}$ & ${ }^{96} \mathrm{Mo}$ & ${ }^{98} \mathrm{Mo}$ & ${ }^{100} \mathrm{Mo}$ \\
\hline 5.0 & $2.57(-1)$ & $4.95(-2)$ & $1.30(-2)$ & - & - \\
10.0 & $1.41(0)$ & $6.30(-1)$ & $3.33(-1)$ & $6.12(-2)$ & $9.88(-3)$ \\
15.0 & $3.82(0)$ & $2.14(0)$ & $1.32(0)$ & $4.08(-1)$ & $1.33(-1)$ \\
20.0 & $8.21(0)$ & $5.14(0)$ & $3.51(0)$ & $1.53(0)$ & $6.91(-1)$ \\
25.0 & $1.56(1)$ & $1.05(1)$ & $7.73(0)$ & $4.18(0)$ & $2.22(0)$ \\
30.0 & $2.68(1)$ & $1.92(1)$ & $1.49(1)$ & $9.22(0)$ & $5.43(0)$ \\
40.0 & $6.52(1)$ & $4.97(1)$ & $4.14(1)$ & $2.97(1)$ & $2.01(1)$ \\
50.0 & $1.33(2)$ & $1.05(2)$ & $9.04(1)$ & $6.94(1)$ & $5.10(1)$ \\
60.0 & $2.44(2)$ & $1.97(2)$ & $1.75(2)$ & $1.39(2)$ & $1.07(2)$ \\
70.0 & $4.00(2)$ & $3.34(2)$ & $3.02(2)$ & $2.50(2)$ & $2.00(2)$ \\
80.0 & $5.95(2)$ & $5.09(2)$ & $4.67(2)$ & $3.99(2)$ & $3.33(2)$ \\
\hline
\end{tabular}

Woods-Saxon potential with the parameters of [27]. For the ${ }^{100}$ Mo nucleus, some of the singleparticle energies close to the respective Fermi surfaces were then adjusted according to [28].

We employ the formalism discussed in Section 2.2 to calculate the double-differential cross sections (2.20) for all the final nuclear states $f$ separately. The total cross section $\sigma\left(E_{\mathbf{k}}\right)$ as a function of the energy of the impinging neutrino $E_{\mathbf{k}}$ then is computed by integrating over the scattering angle $\theta$ and adding up all the individual contributions coming from the final states. For the calculations of the involved nuclear matrix elements, we use the efficient method introduced in [18]. For more details, we refer to [29, 30].

In Table 1 , we show our calculated cross sections $\sigma\left(E_{\mathbf{k}}\right)$ for the charged-current neutrino-nucleus scattering off the even molybdenum isotopes as functions of the energy $E_{\mathrm{k}}$ of the incoming neutrino. In the table, the cross sections are tabulated for a large set of neutrino energies which, are relevant for supernova neutrinos, that is, $E_{\mathbf{k}} \leqslant 80 \mathrm{MeV}$. The results for the antineutrino-induced reactions are similarly shown in Table 2. As is seen in the tables, both the neutrino and antineutrino cross sections increase strongly with increasing neutrino energy. We can also conclude that the neutrino cross sections increase significantly with increasing neutron number $N$. For low energies of the incoming neutrino, the $v$ cross 
sections are dominated by Gamow-Teller-like transitions mediated by the operator $j_{0}(q r) \boldsymbol{\sigma} \boldsymbol{\tau}_{+}$ and Fermi-like ones, which proceed via the operator $j_{0}(q r) \boldsymbol{\tau}_{+}$. At zero momentum transfer $(q=0)$, these operators reduce to the usual Gamow-Teller $\left(\boldsymbol{\sigma} \boldsymbol{\tau}_{+}\right)$and Fermi $\left(\boldsymbol{\tau}_{+}\right)$operators. It is well known that the Gamow-Teller operator obeys the Ikeda sum rule [31]

$$
S_{1^{+}}\left(\beta^{-}\right)-S_{1^{+}}\left(\beta^{+}\right)=3(N-Z)
$$

where $S_{1^{+}}\left(\beta^{-}\right)\left(S_{1^{+}}\left(\beta^{+}\right)\right)$is the total Gamow-Teller strength for the $\beta^{-}\left(\beta^{+}\right)$channel. The nuclei in this work have large neutron excess and hence the $\beta^{+}$branch is small and therefore $S_{1^{+}}\left(\beta^{-}\right) \approx 3(N-Z)$. Similarly, for the transitions to $0^{+}$final states one has that $S_{0^{+}}\left(\beta^{-}\right) \approx N-Z$. Furthermore, the energy threshold, $Q_{\mathrm{EC}}$, is decreasing from $7.870 \mathrm{MeV}\left({ }^{92} \mathrm{Mo}\right)$ to $0.168 \mathrm{MeV}$ $\left({ }^{100} \mathrm{Mo}\right)$. This explains the increase of the neutrino cross sections with increasing neutron number at low neutrino energies. Contrary to this, the antineutrino cross sections decrease with increasing $N$. This is explained by the fact that the $Q_{\beta^{-}}$values are increasing significantly with increasing neutron number.

In the last column of Table 1, we compare our results for ${ }^{100} \mathrm{Mo}$ with those of [10]. It is seen in the table that our results agree well with the ones published in the aforementioned reference. Our computed neutrino cross sections for ${ }^{100} \mathrm{Mo}$ are also in qualitative agreement with the results displayed in Figure 1 of [14].

As already mentioned above, we use in the present calculations the method of barycentric Lagrange interpolation for the computations of the required nuclear matrix elements. Therefore, in Figure 2 we compare the cross sections computed with this method with exact calculations in the case of the neutrino scattering off ${ }^{100} \mathrm{Mo}$. The results for the antineutrino reaction are similarly shown in Figure 3. It is seen in the figures that the results computed by the approximate method (based on numerical interpolation) are accurate to 4-5 significant digits. Consequently, the numerical errors are very small compared to the errors coming from, for example, uncertainties in the nuclear wave functions. The results for the other isotopes are similar and are thus not shown here.

From the experimental point-of-view, an interesting quantity is the flux-averaged cross section $\langle\sigma\rangle$, which is obtained by folding the cross sections $\sigma\left(E_{\mathbf{k}}\right)$ with an appropriate energy profile of the incoming neutrinos. In this work, we adopt a two-parameter Fermi-Dirac distribution

$$
n_{\mathrm{FD}}\left(E_{\mathbf{k}}\right)=\frac{1}{T_{v}^{3} F_{2}\left(\alpha_{v}\right)} \frac{E_{\mathbf{k}}^{2}}{\exp \left(E_{\mathbf{k}} / T_{v}-\alpha_{v}\right)+1},
$$

where $T_{v}$ denotes the effective neutrino temperature and $\alpha_{v}$ is the so-called pinching parameter. Here the constant $F_{2}\left(\alpha_{v}\right)$ normalizes the total flux to unity.

Because of interactions with the matter, the neutrinos undergo flavor conversions when they propagate through the star (see e.g., [32]). Consequently, the energy profiles of the neutrinos (or antineutrinos) which arrive at the Earth-bound detector are different from the initial ones. For simplicity, we assume in this work that the oscillations occur in the outer layers of the star. Furthermore, we neglect collective neutrino-neutrino interactions. 


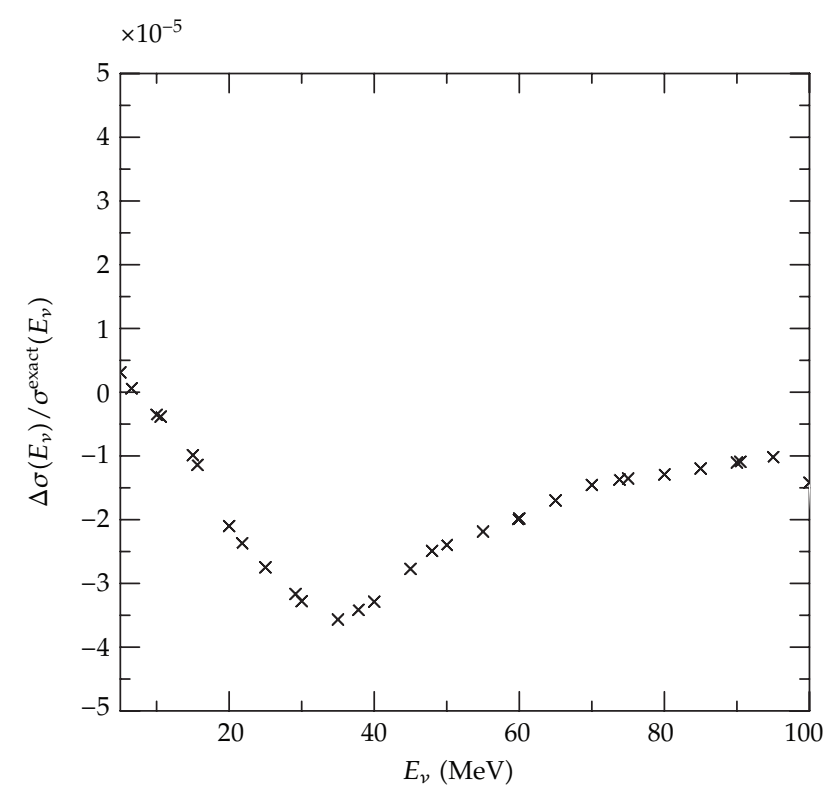

Figure 2: Numerical errors in the cross section for the charged-current neutrino-nucleus scattering off ${ }^{100}$ Mo due to the use of barycentric Lagrange interpolation. The errors are shown for various energies $E_{v}$ of the incoming neutrino. In the figure, $\Delta \sigma\left(E_{v}\right)=\sigma^{\text {approx }}\left(E_{v}\right)-\sigma^{\text {exact }}\left(E_{v}\right)$.

The neutrino profiles for the neutrinos, which reach the detector can then be written in the forms [32]

$$
\begin{aligned}
& F_{v_{\mathrm{e}}}= \begin{cases}\sin ^{2} \theta_{13} F_{v_{\mathrm{e}}}^{0}+\cos ^{2} \theta_{13} F_{v_{x}}^{0} & \text { Normal hierarchy, } \\
\sin ^{2} \theta_{12} F_{v_{\mathrm{e}}}^{0}+\cos ^{2} \theta_{12} F_{v_{x}}^{0} & \text { Inverted hierarchy, }\end{cases} \\
& F_{\bar{v}_{\mathrm{e}}}= \begin{cases}\cos ^{2} \theta_{12} F_{\bar{v}_{\mathrm{e}}}^{0}+\sin ^{2} \theta_{12} F_{\bar{v}_{x}}^{0} & \text { Normal hierarchy, } \\
\sin ^{2} \theta_{13} F_{\bar{v}_{\mathrm{e}}}^{0}+\cos ^{2} \theta_{13} F_{\bar{v}_{x}}^{0} & \text { Inverted hierarchy, }\end{cases}
\end{aligned}
$$

for neutrinos and antineutrinos, respectively. Here $F_{v_{\mathrm{e}}}^{0}\left(F_{v_{x}}^{0}\right)$ is the energy profile of electron neutrinos (non-electron neutrinos) and correspondingly for the antineutrinos. We also point out that due to the large rest masses of the muon and tau (and their antiparticles), only supernova $v_{\mathrm{e}}$ and $\bar{v}_{\mathrm{e}}$ can be detected by charged-current neutrino-nucleus scattering.

In Table 3 are shown the computed averaged cross sections for the supernova $v$ and $\bar{v}$ induced scatterings off ${ }^{100} \mathrm{Mo}$. In the table, $v_{\mathrm{e}}\left(\bar{v}_{\mathrm{e}}\right)$ represents nonoscillating electron neutrinos (electron antineutrinos). We moreover denote with $v_{\mathrm{ex}}^{\mathrm{NH}}\left(\bar{v}_{\mathrm{ex}}^{\mathrm{NH}}\right)$ and $v_{\mathrm{ex}}^{\mathrm{IH}}\left(\bar{v}_{\mathrm{ex}}^{\mathrm{IH}}\right)$ the oscillating electron neutrinos (electron antineutrinos) for the normal and inverted hierarchies, respectively. According to (3.3) (see (3.4)), these neutrinos have energy profiles that are altered with respect to the initial ones by the flavor conversions $v_{x} \rightarrow v_{\mathrm{e}}$. Here results are shown for the two sets of neutrino parameters shown in Table 4. Averaged cross sections for other energy profiles of the incoming neutrinos (antineutrinos) can be easily computed by using the original cross sections tabulated in Table 1 (Table 2). We conclude from Table 3 that the flavor transformations significantly increase the averaged cross sections both for neutrinos and antineutrinos. The antineutrino cross sections are also much smaller 


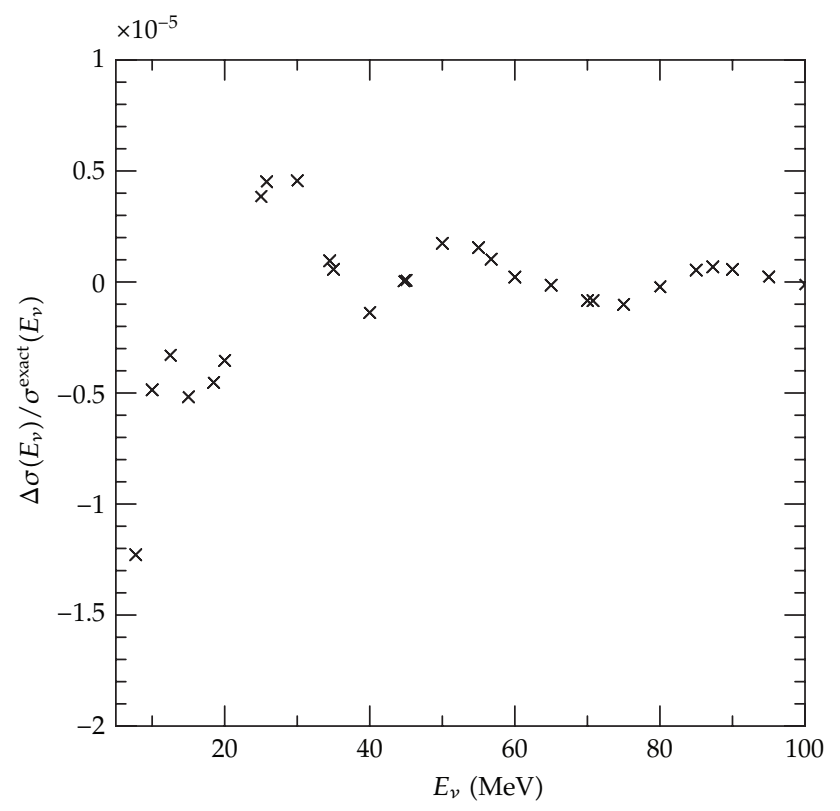

Figure 3: Same as Figure 2 for the charged-current antineutrino scattering off ${ }^{100} \mathrm{Mo}$.

Table 3: Averaged cross sections for the charged-current neutrino and antineutrino scatterings off ${ }^{100} \mathrm{Mo}$ in units of $10^{-41} \mathrm{~cm}^{2}$ calculated for the two sets of neutrino parameters (I and II) displayed in Table 4. In the table, we also show for comparison the results computed in [33].

\begin{tabular}{lcccccc}
\hline & $v_{\mathrm{e}}$ & $v_{\mathrm{ex}}^{\mathrm{NH}}$ & $v_{\mathrm{ex}}^{\mathrm{IH}}$ & $\bar{v}_{\mathrm{e}}$ & $\bar{v}_{\mathrm{ex}}^{\mathrm{NH}}$ & $\bar{v}_{\mathrm{ex}}^{\mathrm{IH}}$ \\
\hline pnQRPA (I) & 5.80 & 24.1 & 18.7 & 0.048 & 0.093 & 0.189 \\
pnQRPA (II) & 6.79 & 77.9 & 56.7 & 0.177 & 0.492 & 1.182 \\
Ejiri et al. [33] & 5.84 & 76.5 & & & & \\
\hline
\end{tabular}

than the corresponding ones for neutrinos. This is explained by the large neutron excess for ${ }^{100} \mathrm{Mo}(N-Z=16)$ since then the antineutrino-induced reactions are suppressed because of Pauli blocking. In the table, we also compare our results with the ones of [33] for the parameter set II of Table 4 . The numbers computed in the aforementioned reference have here been transformed to take into account the oscillation effect (3.3) with the updated value of $\sin ^{2} \theta_{13}=0.025$ [34] adopted in the present calculations. The discrepancy between the two calculations for $v_{\mathrm{e}}$ is about $16 \%$ and for the flavor converted neutrinos in the normal hierarchy the results are almost exactly the same. Here it should be noted that in the aforementioned reference the measured $0^{-}, 1^{+}$, and $2^{-}$strength distributions of [35] were adopted instead of those calculated by the use of the pnQRPA. We therefore conclude that the results are in very good agreement with each other despite the rather different methods used in the calculations.

The proposed MOON detector can be realized by either using enriched ${ }^{100}$ Mo or natural molybdenum [33,36]. Consequently, accurate estimates of the nuclear responses for all the stable molybdenum isotopes are important. Therefore, we show in Table 5 our calculated averaged cross sections for all the stable even-even $(A=92,94,96,98,100)$ Mo nuclei. It is seen in the table that the values of the neutrino cross sections are increasing significantly with increasing neutron number, while for the antineutrino reaction (2.10) the trend is opposite. We also conclude again that the cross sections for the neutrino scattering are 
Table 4: Average neutrino energies and values of the parameter $\alpha$ for the two sets of neutrino parameters adopted in the present calculations. In the table, $x$ denotes the nonelectron flavors, that is, $x=\mu, \tau$.

\begin{tabular}{lcccc}
\hline Parameter set & $\left(\left\langle E_{v_{\mathrm{e}}}\right\rangle, \alpha_{v_{\mathrm{e}}}\right)$ & $\left(\left\langle E_{\bar{v}_{\mathrm{e}}}\right\rangle, \alpha_{\bar{v}_{\mathrm{e}}}\right)$ & $\left(\left\langle E_{v_{x}}\right\rangle, \alpha_{v_{x}}\right)$ & $\left(\left\langle E_{\bar{v}_{x}}\right\rangle, \alpha_{\bar{v}_{x}}\right)$ \\
\hline I & $(11.5,3.0)$ & $(13.6,3.0)$ & $(16.3,0.0)$ & $(16.3,0.0)$ \\
II & $(11.0,0.0)$ & $(16.0,0.0)$ & $(25.0,0.0)$ & $(25.0,0.0)$ \\
\hline
\end{tabular}

Table 5: Averaged cross sections for the charged-current neutrino-nucleus scattering from the even Mo isotopes in units of $10^{-41} \mathrm{~cm}^{2}$ computed with the neutrino parameters corresponding to set I of Table 4 .

\begin{tabular}{lcccccc}
\hline Nucleus & $v_{e}$ & $v_{\mathrm{ex}}^{\mathrm{NH}}$ & $v_{\mathrm{ex}}^{\mathrm{IH}}$ & $\bar{v}_{e}$ & $\bar{v}_{\mathrm{ex}}^{\mathrm{NH}}$ & $\bar{v}_{\mathrm{ex}}^{\mathrm{IH}}$ \\
\hline${ }^{92} \mathrm{Mo}$ & 1.30 & 8.74 & 6.54 & 0.457 & 0.606 & 0.934 \\
${ }^{94} \mathrm{Mo}$ & 2.19 & 12.2 & 9.18 & 0.283 & 0.396 & 0.645 \\
${ }^{96} \mathrm{Mo}$ & 3.16 & 15.6 & 11.9 & 0.196 & 0.290 & 0.495 \\
${ }^{98} \mathrm{Mo}$ & 4.20 & 18.6 & 14.8 & 0.094 & 0.160 & 0.304 \\
${ }^{100} \mathrm{Mo}$ & 6.41 & 25.7 & 20.0 & 0.048 & 0.093 & 0.189 \\
\hline
\end{tabular}

notably larger than the ones for the antineutrino channel. Computations of the cross sections of the charged-current neutrino-nucleus scattering off the odd isotopes, ${ }^{95} \mathrm{Mo}$ and ${ }^{97} \mathrm{Mo}$, are still in progress and the results will be published elsewhere.

\section{Conclusions}

In this work, we have computed the cross sections for the charged-current neutrino and antineutrino scatterings off the stable molybdenum isotopes for neutrino energies, which are relevant for supernova neutrinos. In the calculations, the initial and final nuclear states have been constructed by using the pnQRPA. We have tabulated the cross sections for an extensive set of neutrino energies. The nuclear responses for the aforementioned nuclei have subsequently been calculated by using a two-parameter Fermi-Dirac distribution. However, averaged cross sections for other energy profiles of the incoming neutrino (or antineutrino) can be easily computed by using the cross sections given in this paper.

Our results for the neutrino-nucleus scattering off ${ }^{100} \mathrm{Mo}$ agree well with previously published results. We have found for the studied chain of nuclei that the neutrino-scattering cross sections increase significantly with the neutron number $N$. Contrary to this, the cross sections for the antineutrino scattering decrease notably with increasing neutron number.

\section{Acknowledgments}

This work was supported by the Academy of Finland under the Finnish Center Of Excellence Program 2012-2017 (Nuclear and Accelerator Based Program at JYFL). Dr. E. Ydrefors would like to thank Professor H. Ejiri for fruitful discussions.

\section{References}

[1] C. Volpe, "Neutrino-nucleus interactions: open questions and future projects," Nuclear Physics B. Proceedings Supplements, vol. 143, pp. 43-50, 2005.

[2] C. Volpe, "What about a beta-beam facility for low-energy neutrinos?" Journal of Physics G, vol. 30, no. 7, p. L1, 2004. 
[3] H.-T. Janka, K. Langanke, A. Marek, G. Martínez-Pinedo, and B. Müller, "Theory of core-collapse supernovae," Physics Reports, vol. 442, no. 1-6, pp. 38-74, 2007.

[4] H. T. Janka, "Explosion mechanisms of core-collapse supernovae," Annual Review of Nuclear and Particle Science, vol. 62, pp. 407-451, 2012, http:/ / arxiv.org/abs/1206.2503.

[5] C. Giunti and C. W. Kim, Fundamentals of Neutrino Physics and Astrophysics, Oxford University Press, New York, NY, USA, 2007.

[6] G. G. Raffelt, "Physics opportunities with supernova neutrinos," Progress in Particle and Nuclear Physics, vol. 64, no. 2, pp. 393-399, 2010.

[7] P. D. Serpico, S. Chakraborty, T. Fischer, L. Hüdepohl, H.-T. Janka, and A. Mirizzi, "Probing the neutrino mass hierarchy with the rise time of a supernova burst," Physical Review D, vol. 85, Article ID 085031, 13 pages, 2012.

[8] South Pole Neutrino Observatory, http:/ /icecube.wisc.edu/.

[9] C. Volpe, "Neutrino-nucleus interactions as a probe to constrain double-beta decay predictions ," Journal of Physics G, vol. 31, no. 8, p. 903, 2005.

[10] R. Lazauskas and C. Volpe, "Neutrino beams as a probe of the nuclear isospin and spin-isospin excitations," Nuclear Physics A, vol. 792, no. 3-4, pp. 219-228, 2007.

[11] J. Suhonen and O. Civitarese, "Weak-interaction and nuclear-structure aspects of nuclear double beta decay," Physics Report, vol. 300, no. 3-4, pp. 123-214, 1998.

[12] H. Ejiri, "Nuclear spin isospin responses for low-energy neutrinos ," Physics Reports, vol. 338, no. 3, pp. 265-351, 2000.

[13] K. Scholberg, "Supernova neutrino detection," Annual Review of Nuclear and Particle Science, vol. 62, pp. 81-103, 2012.

[14] H. Ejiri, P. Doe, S. R. Elliott et al., "MOON for neutrino-less double beta decays," European Physical Journal: Special Topics, vol. 162, no. 1, pp. 239-250, 2008.

[15] J. S. O'Connell, T. W. Donnelly, and J. D. Walecka, “Semileptonic weak interactions with $C^{12}$," Physical Review C, vol. 6, no. 3, pp. 719-733, 1972.

[16] J. D. Walecka, Theoretical Nuclear and Subnuclear Physics, Imperial College Press, London, UK, 2004.

[17] J. Suhonen, From Nucleons to Nucleus: Concepts of Microscopic Nuclear Theory, Theoretical and Mathematical Physics, Springer, Berlin, Germany, 2007.

[18] E. Ydrefors, K. G. Balasi, T. S. Kosmas, and J. Suhonen, "The response of ${ }^{95,97}$ Mo to supernova neutrinos," Nuclear Physics A, vol. 866, no. 1, pp. 67-78, 2011.

[19] J.-P. Berrut and L. N. Trefethen, "Barycentric lagrange interpolation," SIAM Review, vol. 46, no. 3, pp. 501-517, 2004.

[20] J. Suhonen, T. Taigel, and A. Faessler, "pnQRPA calculation of the $\beta^{+} / \mathrm{EC}$ quenching for several neutron-deficient nuclei in mass regions $A=94-110$ and $A=146-156$ \{black star\}," Nuclear Physics, Section A, vol. 486, no. 1, pp. 91-117, 1988.

[21] J. Suhonen, "Calculation of allowed and first-forbidden beta-decay transitions of odd-odd nuclei," Nuclear Physics A, vol. 563, no. 2, pp. 205-224, 1993.

[22] E. Ydrefors, K. G. Balasi, J. Suhonen, and T. S. Kosmas, “Nuclear responses to supernova neutrinos for the stable molybdenum isotopes," in Neutrinos: Properties, Reactions, Sources and Detection, J. P. Greene, Ed., p. 151, Nova Science, 2011.

[23] W. M. Alberico, S. M. Bilenky, and C. Maieron, "Strangeness in the nucleon: neutrino-nucleon and polarized electron-nucleon scattering," Physics Reports, vol. 358, no. 4, pp. 227-308, 2002.

[24] E. Kolbe, K. Langanke, G. Martínez-Pinedo, and P. Vogel, "Neutrino-nucleus reactions and nuclear structure," Journal of Physics G, vol. 29, no. 11, pp. 2569-2596, 2003.

[25] J. Engel, "Approximate treatment of lepton distortion in charged-current neutrino scattering from nuclei ," Physical Review C, vol. 57, pp. 2004-2009, 1998.

[26] H. Behrens and W. Buhring, Electron Radial Wave Functions and Nuclear Beta Decay, Clarendon, Oxford, $\mathrm{UK}, 1982$.

[27] A. Bohr and B. R. Mottelson, Nuclear Structure, vol. 1, Benjamin, New York, NY, USA, 1969.

[28] J. Suhonen, "Opening of the $Z=40$ subshell gap and the double-beta decay of ${ }^{100} \mathrm{Mo}$," Nuclear Physics A, vol. 700, no. 1-2, pp. 649-665, 2002.

[29] E. Ydrefors, K. G. Balasi, T. S. Kosmas, and J. Suhonen, "The response of ${ }^{95,97}$ Mo to supernova neutrinos," Nuclear Physics A, vol. 866, no. 1, pp. 67-78, 2011.

[30] E. Ydrefors, K. G. Balasi, T. S. Kosmas, and J. Suhonen, "Erratum: the response of ${ }^{95,97}$ Mo to supernova neutrinos," Nuclear Physics A, vol. 878, pp. 1-2, 2012.

[31] K. Ikeda, "Collective excitation of unlike pair states in heavier nuclei," Progress of Theoretical Physics, vol. 31, no. 3, pp. 434-451, 1964. 
[32] A. S. Dighe and A. Y. Smirnov, "Identifying the neutrino mass spectrum from a supernova neutrino burst," Physical Review D, vol. 62, no. 3, Article ID 033007, pp. 1-24, 2000.

[33] H. Ejiri, J. Engel, and N. Kudomi, "Supernova-neutrino studies with ${ }^{100}$ Mo," Physics Letters, Section B, vol. 530, no. 1-4, pp. 27-32, 2002.

[34] D. V. Forero, M. Toero, and J. W. F. Valle, "Global status of neutrino oscillation parameters after Neutrino-2012," Physical Review D, vol. 86, Article ID 073012, 8 pages, 2012.

[35] H. Akimune, H. Ejiri, M. Fujiwara et al., "GT strengths studied by $\left({ }^{3} \mathrm{He}, \mathrm{t}\right)$ reactions and nuclear matrix elements for double beta decays," Physics Letters, Section B, vol. 394, no. 1-2, pp. 23-28, 1997.

[36] H. Ejiri, Private Communication, 2012. 

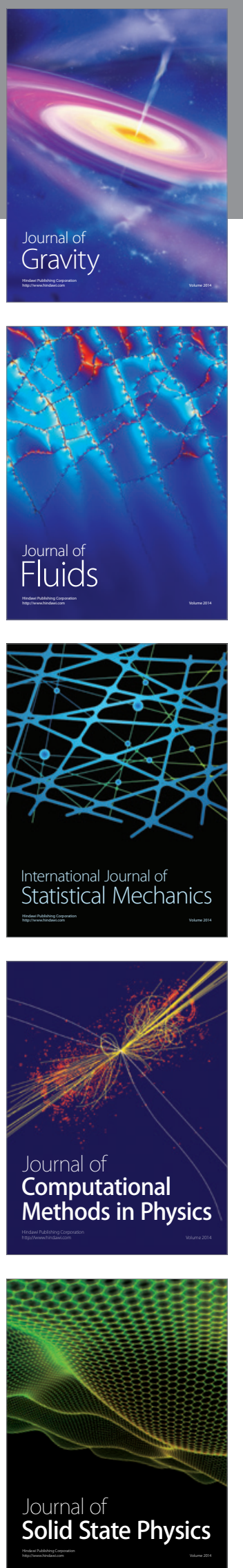

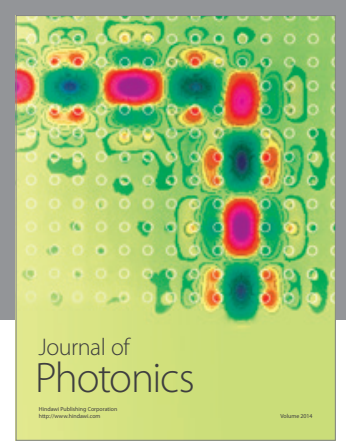

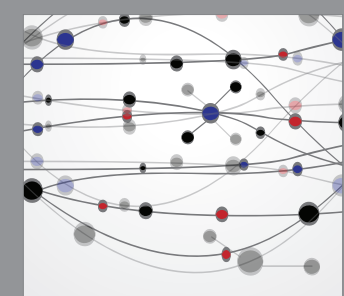

The Scientific World Journal
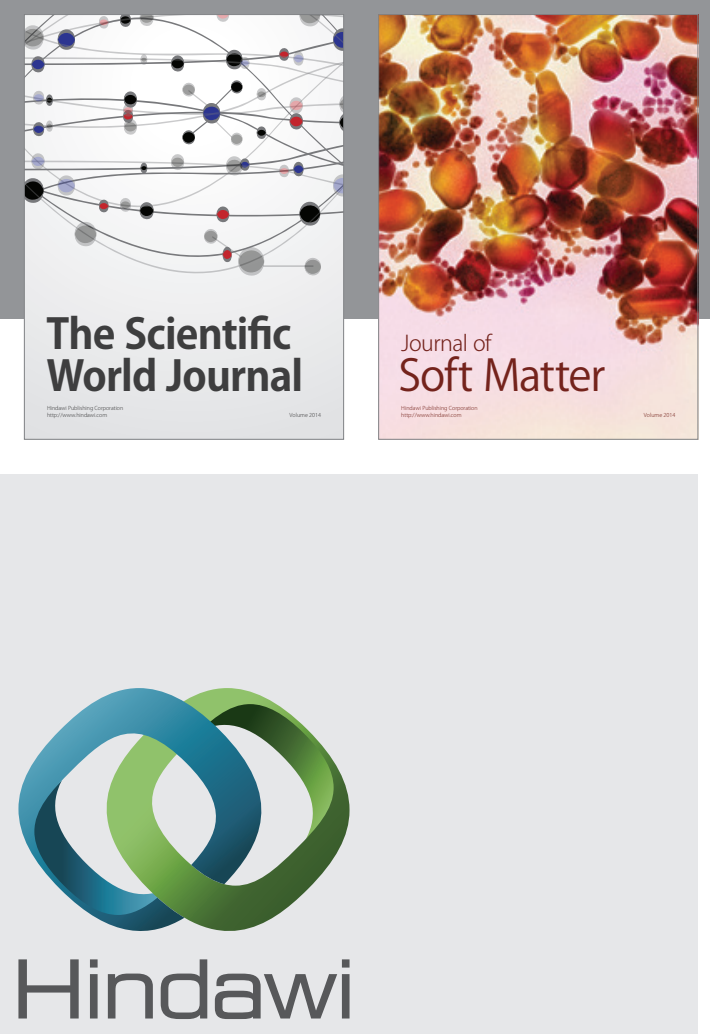

Submit your manuscripts at

http://www.hindawi.com
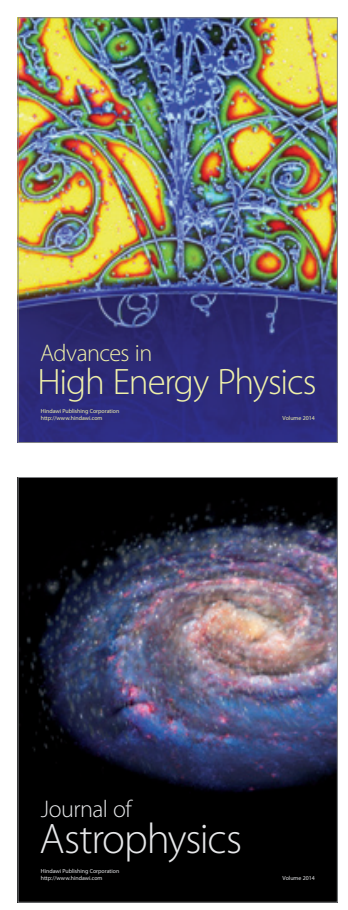
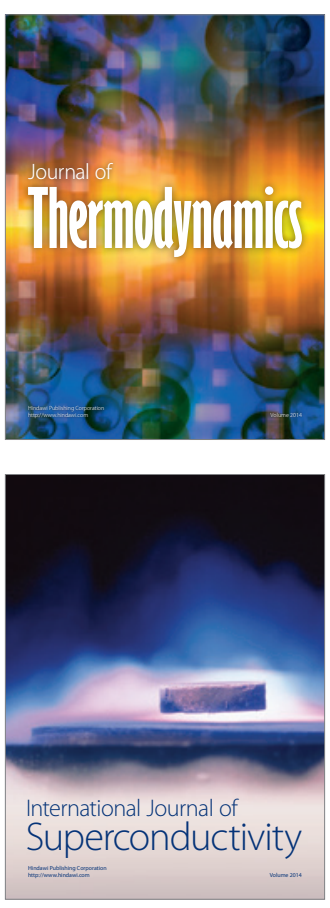
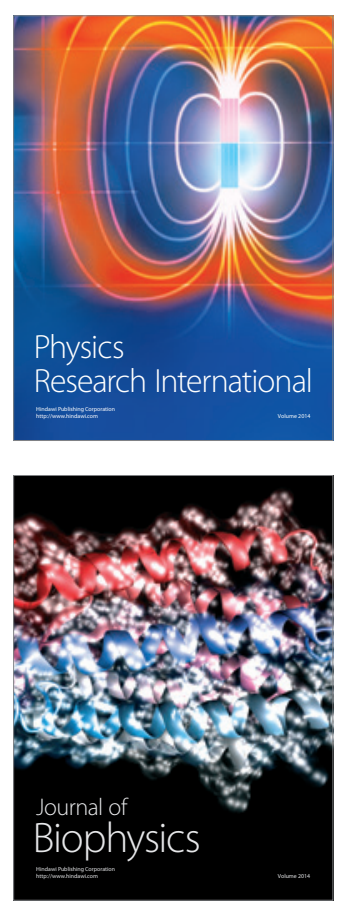
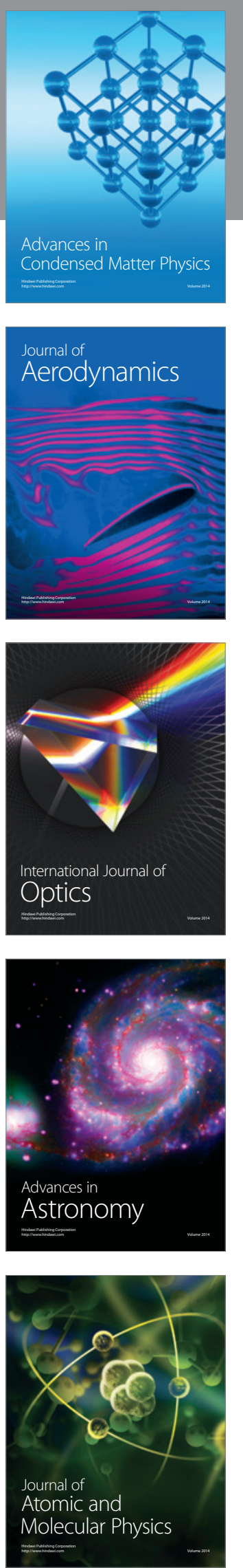